\title{
FUSE Observations of Mass Loss in Planetary Nebulae
}

\author{
Rosina Iping ${ }^{1,2} \&$ George Sonneborn ${ }^{2}$ \\ ${ }^{1}$ Catholic University of America, Washington, DC \\ ${ }^{2} N A S A / G S F C$ Code 681, Greenbelt, MD 20771
}

\begin{abstract}
The Far Ultraviolet Spectroscopic Explorer (FUSE) satellite observed several high and low excitation planetary nebulae in the wavelength range 905-1187 $\AA$ at a spectral resolution of $\lambda / \Delta \lambda \sim 15,000$.
\end{abstract}

Planetary nebulae are a key pathway to return processed material to the ISM. The central star's photospheric continua are uncertain due to nebular continuum emission. The mass-loss rates of the central-star winds can be derived by comparing the observed P-Cygni profiles with theoretical profiles, assuming a velocity law and a ionization structure. We report on the analysis of the interstellar, nebular, and stellar wind features of NGC 7009, NGC 6853, PK 320-09 and IC2149.

The high-excitation planetary nebula NGC 7009 and its central star were observed by the FUSE satellite (Moos et al. 2000, ApJL 538, L1) using the $30 \times 30$ arcsec aperture. Although IUE spectra show that stellar C IV 1550 is absent and N V 1240 is weak, the FUSE spectrum reveals a very strong O VI 1032-38 P-Cygni line profile with a terminal velocity of $\sim 2400 \mathrm{~km} / \mathrm{sec}$. Nebular emission is also present in He II, C III, N II, N III, S III, S IV, and several unidentified features. Molecular hydrogen absorption appears to be interstellar in origin (see Figure 1a).

Observations of the central stars of NGC 6853 and PK 320-09 (He 2-138) show two absorption components in molecular hydrogen, one interstellar and one probably nebular. For PK 320-09 1, the two components are separated by $\sim 50 \mathrm{~km} / \mathrm{s}$, with the blue shifted component at $\sim-50 \mathrm{~km} / \mathrm{s}$ corresponding with the radial velocity of the PN (see Figure 1b). The populations of different rotational levels of $\mathrm{H}_{2}$ show that the main ISM component is present up to $\mathrm{J}=3$ or 4 , while the blue-shifted component is present up to $\mathrm{J}=5$ or 6 . Comparison with atomic lines (e.g. Fe II) shows that the blue-shifted component is very weak (see Sonneborn, these proceedings). The origin of the nebular $\mathrm{H}_{2}$ remains an open question. Was it formed in the atmosphere of the star before the nebular ejection, or in the nebular gas on newly-formed dust grains? FUSE observations of PNe may shed new light on this question.

FUSE observations of IC 2149 indicate an equatorial ring and a polar component, indicating beamed mass outflow. IC 2149 has a very rich stellar wind spectrum, with lines of C III, Si IV, S III, S IV, P V, and O VI showing P-Cygni profiles. Importantly, most of these lines are unsaturated. They also have very different shape profiles, consistent with the picture that we are seeing an asym- 

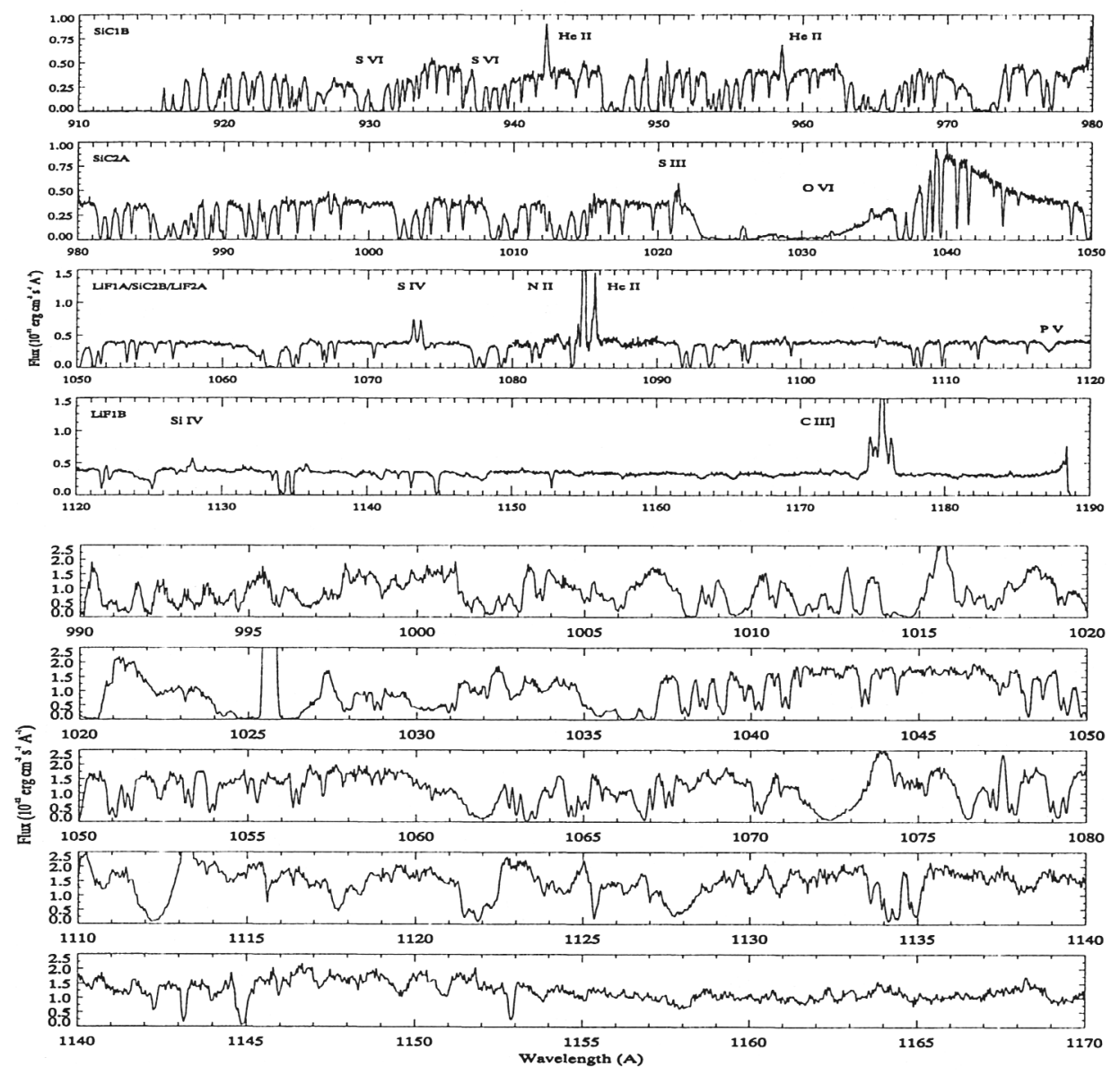

Figure 1. FUSE spectra of NGC 7009 (top) and PK 320-09 (bottom).

metric wind. Aspherical mass loss is thought to produce the bipolar structures seen in so many PNe.

Acknowledgments. This work is based on data obtained for the FUSE Guaranteed Time Team by the NASA-CNES-CSA FUSE mission operated by the Johns Hopkins University. Financial support has been provided in part by NASA contract NAS5-32985 to JHU and NASA grant NAG5-8631 to CUA.

\section{References}

Moos et al. 2000, ApJL 538, L1 